\title{
SOME RANDOM FIXED POINT THEOREMS FOR CONDENSING AND NONEXPANSIVE OPERATORS
}

\author{
HONG-KUN XU
}

(Communicated by Palle E. T. Jorgensen)

\begin{abstract}
Some random versions of deterministic fixed point theorems for condensing and nonexpansive operators are obtained.
\end{abstract}

\section{INTRODUCTION}

Since Bharucha-Reid [1] proved the stochastic version of the well-known Schauder's fixed point theorem, random fixed point theory and applications have been developed rapidly in recent years, see, e.g., Bharucha-Reid [1, 2], Itoh [7, 8], Papageorgiou [12], Sehgal and Singh [15], Sehgal and Waters [16], and Lin [11]. The purpose of the present paper is to continue discussions of this line, that is, some random versions of deterministic fixed point theorems for condensing and nonexpansive operators are derived.

\section{Preliminaries}

Throughout this paper, $(\Omega, \Sigma)$ denotes a measurable space with $\Sigma$ a $\sigma$ algebra of subsets of $\Omega$. For a metric space $(X, d)$, we denote by $C B(X)$ and $(K(X))$ all nonempty closed bounded (compact) subsets of $X$, by $H$ the Hausdorff metric on $C B(X)$ induced by $d$. A multifunction $f: \Omega \rightarrow X$ is called $\left(\Sigma\right.$-)measurable if, for any open subset $B$ of $X, f^{-1}(B):=\{\omega \in \Omega: f(\omega) \cap B \neq$ $\varnothing\} \in \Sigma$. Note that in Himmelberg [6] this is called weakly measurable; since in this paper we use only this type of measurability, we omit the term "weakly" for simplicity. Note also that if $f(\omega) \in K(X)$ for every $\omega \in \Omega$, then $f$ is measurable if and only if $f^{-1}(F) \in \Sigma$ for every closed subset $F$ of $X$. A measurable operator $x: \Omega \rightarrow X$ is called a measurable selector of a measurable multifunction $f: \Omega \rightarrow X$ if $x(\omega) \in f(\omega)$ for each $\omega \in \Omega$. Let $M$ be a nonempty closed subset of $X$. Then a mapping $f: \Omega \times M \rightarrow X$ is called a random operator if, for each fixed $x$ in $M$, the map $f(\cdot, x): \Omega \rightarrow X$ is measurable. A measurable operator $x: \Omega \rightarrow X$ is said to be a random fixed point

Received by the editors June 25, 1989.

1980 Mathematics Subject Classification (1985 Revision). Primary 47H10; Secondary 60H 25.

Key words and phrases. Random fixed point, condensing random operator, nonexpansive random operator, weak inward condition, Leray-Schauder condition. 
of a random operator $f: \Omega \times M \rightarrow X$ if $x(\omega) \in M$ and $f(\omega, x(\omega))=x(\omega)$ for all $\omega \in \Omega$.

If $C$ is a closed subset of a Banach space $X$, a mapping $f: C \rightarrow X$ is called a contraction if there exists a constant $k$ in $(0,1)$ such that

$$
\|f(x)-f(y)\| \leq k\|x-y\|, x, y \in C .
$$

If (1.1) holds true when $k=1, f$ is called nonexpansive. A mapping $f: C \rightarrow$ $X$ is said to be weakly inward [5, p. 55] if $f(x) \in \operatorname{cl} I_{C}(x)$ for all $x \in C$, where cl denotes (norm) closure and

$$
I_{C}(x)=\{z \in X: z=x+a(y-x) \text { for some } y \in C \text { and } a \geq 0\} .
$$

When $C$ has a nonempty interior, a map $f: C \rightarrow X$ is said to satisfy the Leray-Schauder condition if there is a point $w$ in $\operatorname{int}(C)$ such that

$$
f(y)-w \neq m(y-w)
$$

for all $y \in \operatorname{bdy}(C)$, the boundary of $C$, and $m>1$. If $f$ is weakly inward, then it satisfies the Leray-Schauder condition. We recall that a mapping $f: C \rightarrow X$ is said to be demiclosed at $y \in X$ if, for any sequence $\left\{x_{n}\right\}$ in $C$, the conditions $x_{n} \rightarrow x \in C$ weakly and $f\left(x_{n}\right) \rightarrow y$ strongly imply $f(x)=y$. It is well known that (Browder [3]) if $C$ is a bounded closed convex subset of a uniformly convex Banach space and $f: C \rightarrow X$ is nonexpansive, then $I-f$ is demiclosed at every $y \in X$. For more such mappings see Bruck [4].

Now let $B$ be a nonempty bounded subset of $X$. The Kuratowski's measure of noncompactness of $B$ is defined as the number $\alpha(B)=\inf \{c>0: B$ can be covered by a finite number of sets of diameter $\leq c\}$. A mapping $f: C \rightarrow X$ is called condensing if $f$ is continuous and, for each bounded subset $B$ of $C$ with $\alpha(B)>0, \alpha(f(B))<\alpha(B)$. A random operator $f: \Omega \times C \rightarrow X$ is continuous (weakly inward, condensing, nonexpansive, contraction, etc.) if the map $f(\omega, \cdot): C \rightarrow X$ is so, for each fixed $\omega \in \Omega$.

\section{Main Results}

Let $C$ be a nonempty closed bounded convex subset of a reflexive Banach space $X$. Recall that $C$ is said to have the fixed point property (FPP) for nonexpansive mappings if every nonexpansive mapping $T: C \rightarrow C$ has a fixed point (cf. Kirk [9]). Here we say that $C$ has the random fixed point property (RFPP) for nonexpansive random operators if, for any measurable space $(\Omega, \Sigma)$ with $\Sigma$ a sigma algebra of subsets of $\Omega$, every nonexpansive random operator $T: \Omega \times C \rightarrow C$ has a random fixed point. The question now arises as to whether $C$ has the RFPP for nonexpansive random operators if $C$ has the FPP for nonexpansive mappings. In this section, we shall give partial answers to this question. We shall also derive some random fixed point theorems for non-self-condensing and nonexpansive random operators. 
Theorem 1. Let $C$, a nonempty closed bounded convex separable subset of a reflexive Banach space, have the FPP for nonexpansive mappings and let $T: \Omega \times$ $C \rightarrow C$ be a nonexpansive random operator. Suppose one of the following two conditions is satisfied:

(i) $\Sigma$ is closed under the Suslin operation (cf. [17]);

(ii) $X$ is strictly convex and $I-T$ is demiclosed at zero.

Then $T$ has a random fixed point.

Proof. Since $C$ has the FPP for nonexpansive mappings, for each $\omega \in \Omega$, the set

$$
F(\omega):=\{x \in C: T(\omega, x)=x\}
$$

is nonempty and closed. Suppose, first, assumption (i) is satisfied. Then all different definitions of measurability in the literature are equivalent (Wagner [17, Theorem 4.2]). Thus, the same proof as Lin [11, Lemma 1] yields that $F$ admits a measurable selector $x$ that is a random fixed point of $T$. Suppose now that assumption (ii) is satisfied. Since $X$ is strictly convex, $F(\omega)$ is convex and hence weakly compact. We show that $F$ is $w$-measurable, i.e., for each $x^{*}$ in $X^{*}$, the dual space of $X$, the numerically valued function $x^{*} F$ is measurable. Let

$$
F_{n}(\omega):=\{x \in C:\|T(\omega, x)-x\|<1 / n\}, \quad n=1,2, \ldots
$$

By Itoh [7, Proposition 3], each $F_{n}$ is measurable. Let $d_{w}$ be the metric on $C$ induced by the weak topology (the separability of $C$ implies the weak topology on $C$ is a metric topology,) and let $H_{w}$ be the Hausdorff metric produced by $d_{w}$. We claim that, for each $\omega \in \Omega$,

$$
\lim _{n \rightarrow \infty} H_{w}\left(F_{n}(\omega), F(\omega)\right)=0 .
$$

In fact, since $\cap_{n=1}^{\infty} F_{n}(\omega)=F(\omega)$, the limit in (3.1) exists and we denote it by $h(\omega)$. If $h(\omega)>0$, then there exists, for each $n$, an $x_{n}$ in $F_{n}(\omega)$ such that

$$
d_{w}\left(x_{n}, F(\omega)\right)>\frac{1}{2} h(\omega) .
$$

Let $\left\{x_{k^{\prime}}\right\}$ be a subsequence of $\left\{x_{n}\right\}$ converging weakly to some $x \in C$, i.e., $d_{w}\left(x_{k^{\prime}}, x\right) \rightarrow 0$ as $k^{\prime} \rightarrow \infty$. Then (3.2) implies

$$
d_{w}(x, F(\omega)) \geq \frac{1}{2} h(\omega)>0 .
$$

On the other hand, since $\left\|x_{k^{\prime}}-T\left(\omega, x_{k^{\prime}}\right)\right\| \leq 1 / k^{\prime}$ and $I-T(w, \cdot)$ is demiclosed at zero, it follows that $x-T(\omega, x)=0$, i.e., $x \in F(\omega)$. This contradicts (3.3), and (3.1) is proven. Now, by Itoh [7, Proposition 1], $F$ is $w$-measurable. Thus, by Kuratowski and Ryll-Nardzewski [10], there exists a $w$-measurable selector $x$ of $F$, i.e., for each $x^{*} \in X^{*}, x^{*} x$ is measurable as a numericallyvalued function on $\Omega$. Since $C$ is separable, $x$ is measurable [2, Theorem 1.2]. This $x$ is the desired random fixed point of $T$.

Remark 1. If $X$ is uniformly convex, $I-T$ is demiclosed at every $y \in X$ (Browder [3]). Hence the assumption that $\Sigma$ is closed under the Suslin operation in Lin [11, Lemma 1] is superfluous. However, we do not know if this is 
valid in general. We also remark that the boundedness of $f(\omega, S)$ in Lin [11, Lemma 1] can be replaced by the boundedness of $f(\omega, x)$ for some $x \in C$ and each $\omega \in \Omega$, since if $f(\omega, x)$ is bounded for some $x \in C$ and all $\omega \in \Omega$, one can construct for each $\omega \in \Omega$ a closed bounded convex subset $C(\omega)$ of $S$ containing the trajectory $\left\{f^{n}(\omega, x)\right\}$ of $f(\omega, \cdot)$ at $x$ such that $C(\omega)$ is $f(\omega, \cdot)$-invariant, i.e., $f(\omega, C(\omega)) \subseteq C(\omega)$.

We now turn to consider non-self-operators.

Theorem 2. Let $C$ be a nonempty closed convex subset of a separable Banach space $X, T: \Omega \times C \rightarrow X$ a condensing random operator that is either (i) weakly inward or (ii) satisfies the Leray-Schauder condition. Suppose, for each $\omega \in$ $\Omega, T(\omega, C)$ is bounded. Then $T$ has a random fixed point.

Proof. By results of Reich [13, 14], in both cases, the set

$$
F(\omega):=\{x \in C: T(\omega, x)=x\}
$$

is nonempty and closed. Since $T(\omega, F(\omega))=F(\omega)$, it follows from condensingness of $T$ that $\alpha(F(\omega))=0$, i.e., $F$ is compact-valued. Thus, to show the measurability of $F$, it is sufficient to show that, for any closed subset $D$ of $X$, $F^{-1}(D)$ is measurable. Take a countable dense subset $\left\{x_{n}\right\}$ of $C$ and let

$$
L(D)=\bigcap_{n=1}^{\infty} \bigcup_{x_{i} \in D_{n}}\left\{\omega \in \Omega:\left\|T\left(\omega, x_{i}\right)-x_{i}\right\|<1 / n\right\},
$$

where $D_{n}=\{x \in C: d(x, D)<1 / n\}$ and $d(x, D)=\inf \{\|x-y\|: y \in D\}$. As in Itoh $\left[8\right.$, p. 263], one can easily check $F^{-1}(D)=L(D)$. Hence $F$ is measurable. By Kuratowski and Ryll-Nardzewski [10], $F$ has a measurable selector $x: \Omega \rightarrow C$. This $x$ is the desired random fixed point of $T$.

Remark 2. For self-operators, Theorem 2(i) belongs to Itoh [8, Theorem 2.1]. When $C$ is a closed ball centered at zero, or $X$ is a Hilbert space, Theorem 2(i) belongs to Lin [11, Theorems 4,5$]$.

Theorem 3. Let $C$, a nonempty closed bounded convex subset of a separable Banach space $X$, have the RFPP for nonexpansive random operators, and $T: \Omega \times C \rightarrow X$ a nonexpansive random operators. Suppose $T$ is weakly inward. Then $T$ has a random fixed point.

Proof. For a fixed $\omega \in \Omega$ and $t \in(0,1)$ let $r=t /(1-t)$ and

$$
F_{\omega}=F(\omega, \cdot)=\left(I+r\left(I-T_{\omega}\right)\right)^{-1}
$$

where $T_{\omega}=T(\omega, \cdot)$ and $I$ is the identity operator on $X$. Then is is easy to see that $F_{\omega}$ is a well-defined single-valued nonexpansive operator on its domain $D_{\omega}$. For any fixed $z$ in $C$ define $g: C \rightarrow X$ by $g(x)=t T_{\omega}(x)+(1-t) z$. One easily checks the contraction $g$ is also weakly inward (cf. [5, p. 56]) and hence has a fixed point $x_{t} \in C$. This means $z=(1+r) x_{t}-r T_{\omega} x_{t} \in D_{\omega}$ and $F_{\omega}(z)=x_{t} \in C$. Since $z$ is arbitrary, it follows that $D_{\omega} \supset C$ and $F_{\omega}$ maps $C$ into $C$ itself. Therefore $F: \Omega \times C \rightarrow C$ is a nonexpansive random operator 
and thus has a random fixed point $x$ by assumption of the theorem. This $x$ is clearly also a random fixed point of $T$.

Theorem 4. Let $C$ be a nonempty closed bounded convex subset of a separable uniformly convex Banach space $X$ and let $T: \Omega \times C \rightarrow X$ be a nonexpansive random operator. Suppose $C$ has a nonempty interior and $T$ satisfies the LeraySchauder condition, i.e., for each $\omega \in \Omega$, there exists an element $z \in \operatorname{int}(C)$ ( depending on $\omega$ ) such that

$$
f(\omega, y)-z \neq a(y-z)
$$

for all $y \in \operatorname{bdy}(C)$ and $a>1$. Then $T$ has a random fixed point.

Proof. Let $0<t<1$. For a fixed $\omega \in \Omega$, let $z=z(\omega) \in \operatorname{int}(C)$ satisfy (3.4). Then one easily sees that the contraction $f_{\omega}: C \rightarrow X$, defined by

$$
f_{\omega}(x)=t T(\omega, x)+(1-t) z, \quad x \in C,
$$

satisfies the Leray-Schauder condition. Thus, by Reich [14], $f_{\omega}$ has a unique fixed point $x_{t}(\omega)$. As in the proof of Theorem 1(ii), we see that $x_{t}: \Omega \rightarrow C$ is measurable. It follows that there is a sequence $\left\{x_{n}\right\}$ of measurable operators $x_{n}: \Omega \rightarrow C$ satisfying

$$
\lim _{n \rightarrow \infty}\left\|x_{n}(\omega)-T\left(\omega, x_{n}(\omega)\right)\right\|=0
$$

for each $\omega \in \Omega$. Let $F_{n}(\omega)=\mathrm{w}-\mathrm{cl}\left(x_{i}(\omega): i \geq n\right) \quad$ (w-cl denotes the weak closure) and $F(\omega)=\bigcap_{n=1}^{\infty} F_{n}(\omega)$. Then, as in Itoh [8, p. 265], $F$ is weakly compact valued and has a measurable selector $x$. It remains to show that, for each $\omega \in \Omega, x(\omega)$ is a fixed point of $T(\omega, \cdot)$. Toward this end, we choose, from the definition of $F(\omega)$, a subsequence $\left\{x_{n^{\prime}}(\omega)\right\}$ of $\left\{x_{n}(\omega)\right\}$ converging weakly to $x(\omega)$. Since $I-T(\omega, \cdot)$ is demiclosed at zero, it follows from (3.5) that $x(\omega)=T(\omega, x(\omega))$, as required.

\section{REFERENCES}

1. A. T. Bharucha-Reid, Fixed point theorems in probabilistic analysis, Bull. Amer. Math. Soc. 82 (1976), 641-645.

2. __ Random integral equations, Academic Press, New York and London, 1972.

3. F. E. Browder, Semicontractive and semiaccretive nonlinear mappings in Banach spaces, Bull. Amer. Math. Soc. 74 (1968), 660-665.

4. R. E. Bruch, A simple proof of the mean ergodic theorem for nonlinear contractions in Banach spaces, Israel J. Math. 32 (1979), 107-116.

5. K. Goebel and S. Reich, Uniform convexity, hyperbolic geometry, and nonexpansive mappings, Marcel Dekker, New York, and Basel, 1984.

6. C. J. Himmelberg, Measurable relations, Fund. Math. 87 (1975), 53-72.

7. S. Itoh, A random fixed point theorem for a multivalued contraction mapping, Pacific $\mathbf{J}$. Math. 68 (1977), 85-90.

8. __ Random fixed point theorems with an application to random differential equations in Banach spaces, J. Math. Anal. Appl. 67 (1979), 261-273.

9. W. A. Kirk, Fixed point theory for nonexpansive mappings I, II, Lecture Notes in Math., vol. 886, Springer-Verlag, Berlin and New York, 1981, pp. 484-505; Contemp. Math. 18 (1983), 121-140. 
10. K. Kuratowski and C. Ryll-Nardzewski, A general theorem on selections, Bull. Acad. Polon. Sci. Ser. Math. Astronom. Phys. 13 (1965), 397-403.

11. T. C. Lin, Random approximations and random fixed point theorems for non-self-maps, Proc. Amer. Math. Soc. 103 (1988), 1129-1135.

12. N. S. Papageorgiou, Random fixed point theorems for measurable multifunctions in Banach spaces, Proc. Amer. Math. Soc. 97 (1986), 507-514.

13. S. Reich, Fixed points of condensing functions, J. Math. Anal. Appl. 41 (1973), 460-467.

14. $\ldots$, On fixed point theorems obtained from existence theorems for differential equations, J. Math. Anal. Appl. 54 (1976), 26-36.

15. V. M. Sehgal and S. P. Singh, On random approximations and a random fixed point theorem for set valued mappings, Proc. Amer. Math. Soc. 95 (1985), 91-94.

16. V. M. Sehgal and C. Waters, Some random fixed point theorems for condensing operators, Proc. Amer. Math. Soc. 90 (1984), 425-429.

17. D. H. Wagner, Survey of measurable selection theorems, SIAM J. Control Optim. 15 (1977), 859-903.

Institute of Applied Mathematics, East China University of Chemical Technology, Shanghai 200237, The People's Republic of China 